\title{
Modulation of estrogen receptor a levels by endogenous and exogenous ligands
}

\author{
P. La Rosa, M. Marino, F. Acconcia* \\ Department of Biology, University Roma Tre, Viale Guglielmo Marconi, 446, 1-00146, Rome, Italy \\ *acconcia@uniroma3.it
}

KEY WORDS: 17ß-estradiol, estrogen receptor, turnover, polyphenols, endocrine disruptors

\begin{abstract}
$E R \alpha$ is a ligand-activated transcription factor, member of the nuclear receptor superfamily. Regulation of $E R \alpha$ levels is intrinsically required for its transcriptional activity and thus for the modulation of the physiological actions of the cognate hormone I7/-estradiol (E2). Indeed, $E R \alpha$ exogenous ligands that target this molecular circuitry are used as drugs in clinical practice. Interestingly, some natural and synthetic molecules, which human beings are commonly exposed to, interfere with the endocrine system and operate through $E R \alpha$ by selectively modifying its signalling. In addition, these molecules may also modulate $E R \alpha$ cellular content. Here, we report the recent advances in our understanding of how exogenous $E R \alpha$ ligands impact on receptor levels and change the physiological E2-dipendent modulation of specific cellular function.
\end{abstract}

\section{Introduction}

The Estrogen receptors (i.e., ER $\alpha$ and ER $\beta$ ) are present from lower chordates to mammals and exert their functions by mediating developmental and physiological responses to estrogens and, in particular, to $17 \beta$-estradiol (E2) both in males and females. In addition, to these physiological roles, the ERs are known to play a prominent role in the development and in the progression of several diseases including different kind of cancers (e.g., breast and endometrial cancer) [1].

The ERs belong to the nuclear receptor protein superfamily, share a well conserved structure at six functional domains ( $A$ to $F$ domains) with the $C$ region being the DNA binding domain (DBD) and the $E$ region being the ligand binding domain (LBD) and work as ligand activated transcription factors [1].
The regulation of gene expression occurs trough the ER $\alpha$ recruitment to the target genes and follows two distinct pathways. In the direct genomic pathway, ER $\alpha$ recognizes and binds directly to the estrogen responsive element (ERE) located in the promoter of the E2-target genes. On the contrary, in the indirect genomic pathway, the ER $\alpha$ regulates the gene expression of those genes which do not contain the ERE sequence by associating with other transcription factors (e.g., Sp1 and AP-1). Irrespective of whether the mechanism of gene modulation is direct or indirect, $E R \alpha$ interacts with a complex of proteins, which can work as co-activators or co-repressors thus forming a scaffolding structure for other proteins required for productive gene transcription [1].

Beside these two mechanisms, ER $\alpha$ triggers rapid (i.e., seconds to minutes) responses to E2, which are mediated by a pool of the ER $\alpha$ located at the plasma membrane. These fast E2 effects (i.e., non-genomic mechanism) are independent on ER $\alpha$ transcriptional activity and consist in the activation of several signalling cascades (e.g., PI3KI AKT pathway). Functionally, these rapid pathways are necessary and sufficient for the E2-dependent cellular responses related to cell proliferation, survival and apoptosis [1].

Regulation of $E R \alpha$ levels by the endogenous ligand E2

As for other hormone receptors, E2-induced ER $\alpha$ degradation represents a crucial step in coordinating $E R \alpha$ signalling. In particular, the binding of E2 to the receptor drastically lowers the protein half-life from more than 5 hours to less than 2 hours. As many other short-lived proteins, the major mechanism that regulates $E R \alpha$ degradation is the ubiquitin-proteasome pathway. The ER $\alpha$ is addressed to the proteasome because of the action of protein ligases, which polyubiquitinate the $E R \alpha$. Interestingly, ER $\alpha$ proteasome-mediated degradation also occurs in the absence of E2 and is required for maintaining the physiological steady-state of the receptor $[2,3]$.

Nonetheless, signalling regulation of the ER $\alpha$ turnover is not fully understood and still needs to be clarified. Indeed, there are many evidences that $E R \alpha$ phosphorylation plays a major role in receptor degradation. This modification, which depends on the E2-evoked activation of the rapid phosphorylation cascades (see above) and is required 
for receptor activation, could be a signal for receptor ubiquitination. Degradation of ER $\alpha$ could then follow a cyclic pattern in which the receptor, which becomes phosphorylated after E2 binding, binds DNA, then is ubiquinated and finally degraded by the proteasome [2].

\section{Modification of ER $\alpha$ cellular levels by exogenous ligands}

From a physiological point of view, the regulation of the ER $\alpha$ cellular levels by E2 functions not only as a mechanism to limit the cellular response to the hormone but also as a mean to allow cells to efficiently respond to the environmental E2 fluctuations, thus synchronizing gene transcription [2,3]. It is therefore not surprising that deregulation of this negative feedback circuitry contributes to the progression of $E R \alpha$-positive tumours. Indeed, drugs (e.g., fulvestran and tamoxifen) that interfere with the ER $\alpha$ stability are used as therapeutic tools [4].

Interestingly, our research group has recently demonstrated that diverse natural and synthetic exogenous $E R \alpha$ ligands can differentially modulate receptor activities, thus working as 'mechanism-specific ligands'[5]. In particular, naringenin (Nar), a flavanone found in citrus fruit and in grape juice, affects $E R \alpha$ signalling by decoupling the genomic mechanisms from the rapid ones [5]. On the contrary, the synthetic molecule bisphenol A (BPA) mimics $E 2$ in activating ER $\alpha$ signalling [6].

Therefore, in principle, it is possible that these polyphenols could also affect ER $\alpha$ cellular levels. Furthermore, since it is well known that such exogenous ligands act also as endocrine disruptors, it is critical to understand how the exogenous ligand-dependent modulation of the ER $\alpha$ cellular levels could affect the cellular behaviour per se as well as in response to the endogenous ER $\alpha$ ligand E2.

Preliminary results indicate that acute treatment (0-24 hours) of ductal carcinoma cells (MCF-7) with Nar does not trigger $E R \alpha$ breakdown whereas acute treatment with BPA mimics E2 in inducing receptor degradation. On the contrary, chronic exposure (48 hours) of MCF-7 cells to $\mathrm{Nar}$ or BPA results in an increased $\mathrm{ER} \alpha$ cellular content with respect to the one observed under E2 treatment. Interestingly, the different Nar- and BPA-dependent modulation of $E R \alpha$ cellular levels appears to be due to the differential ability of these two molecules to inhibit (Nar) or activate (BPA) the proteasome-degradation pathway. Nonetheless, whether Nar or BPA impact on $E R \alpha$ cellular content by modulating the receptor mRNA levels can not be excluded and is currently under investigation.

Remarkably, administration of Nar and BPA either before or after E2 treatment also results in an increase in the ER $\alpha$ cellular levels when compared to the E2 treatment alone. These evidence indicate that exposure to endocrine disruptors drastically modifies the ER $\alpha$ cellular content and possibly the cell response to E2 may diverge from the physiological one.

\section{Discussion}

The regulation of ER $\alpha$ levels is a process not completely understood. However, it is critical for $E R \alpha$ transcriptional activity and plays a key role in E2 signalling and thus for E2mediated cellular functions. Therefore, ER $\alpha$ cellular content is finely controlled both at transcriptional and posttranscriptional level [7]. The importance of this molecular circuitry is further witnessed by the fact that some ER $\alpha$ binding molecules, which affect receptor turnover, are also used as drugs in clinical practise [8].

Remarkably, some natural and synthetic molecules, which human beings are commonly exposed to, interfere with the endocrine system and operate through the ER $\alpha$ and $E R \beta$ by selectively modifying the ER signalling and the cellular outcomes [5]. Our preliminary evidence indicate that these substances (i.e., Nar and BPA) act by affecting the ER $\alpha$ turnover and possibly ER $\alpha$ mRNA levels. As a consequence, the exogenous ligand-induced modification of the ER $\alpha$ cellular content may result in a different response to cognate hormone E2. Moreover, whether exogenous ligands affect also the regulation of ER $\beta$ cellular content still remains to be evaluated and requires intense investigation. Nonetheless, understanding how exogenous $E R \alpha$ and ER $\beta$ ligands impact on receptor levels and change the physiological E2-dependent modulation of specific cellular functions is a critical issue given the importance of the ER $\alpha$ and ER $\beta$ signalling in several physiological processes and in turn in many hormone-related pathologies (e.g., breast and colon cancers).

\section{References}

[1] Ascenzi P., Bocedi A., Marino M. 2006. Structure-function relationship of estrogen receptor alpha and beta: impact on human health. Mol.Aspects Med., 27(4): 299-402.

[2] Nawaz Z., O'Malley B.W. 2004. Urban renewal in the nucleus: is protein turnover by proteasomes absolutely required for nuclear receptor-regulated transcription? Mol. Endocrinol., 18: 493-499.

[3] Reid G., Hübner M.R., Métivier R., Brand H., Denger S., Manu D., Beaudouin J., Ellenberg J., Gannon F. 2003 Cyclic, proteasome-mediated turnover of unliganded and liganded ERalpha on responsive promoters is an integral feature of estrogen signalling. Mol. Cell, 11:695-707.

[4] Kumar R. 2003. Another tie that binds the MTA family to breast cancer. Cell, 113: 142-143.

[5] Galluzzo P., Marino M. 2006. Nutritional flavonoids impact on nuclear and extranuclear estrogen receptor activities. Genes Nutr., 1: 161-176.

[6] Ricupito A., Del Pozzo G., Diano N., Grano V., Portaccio M., Marino M., Bolli A., Galluzzo P., Bontempo P., Mita L., Altucci L., Mita D.G. 2009. Effect of bisphenol A with or without enzyme treatment on the proliferation and viability of MCF-7 cells. Environ. Int., 35: 21-26.

[7] Matthews ]., Gustafsson J.A. 2003. Estrogen signaling: a subtle balance between ER alpha and ER beta. Mol. Interv., 3: 281-292

[8] Morgan D.A., Refalo N.A., Cheung K.L. 2011. Strength of ERpositivity in relation to survival in ER-positive breast cancer treated by adjuvant tamoxifen as sole systemic therapy. Breast. 20:215-219. 